\title{
O Desenho da Figura Humana é válido para avaliar ansiedade em crianças? ${ }^{1}$
}

\author{
Denise Ruschel Bandeira² \\ Adriana Loguercio ${ }^{3}$ \\ Wolnei Caumo ${ }^{4}$ \\ Maria Beatriz Cardoso Ferreira ${ }^{5}$
}

\begin{abstract}
Resumo
A ansiedade vem sendo avaliada de diferentes formas, entre elas através do Desenho da Figura Humana (DFH). A principal escala de avaliação do DFH foi idealizada por Handler, possuindo divergências quanto a sua validade entre os pesquisadores. Este trabalho tem por objetivo, então, avaliar a validade desta escala, comparando desenhos de crianças em situação pré-cirúrgica e desenhos realizados por crianças de um grupo controle. Os pontos obtidos nos desenhos também são comparados aos escores no Inventário de Ansiedade Traço-Estado para Crianças (IDA TE-C). Foram investigados desenhos de 106 crianças, de idades entre 7 e 13 anos, de ambos os sexos. Os resultados mostram haver diferenças significativas somente entre as escalas de ansiedade traço-estado entre os grupos controle e experimental. Os índices de ansiedade de Handler não evidenciaram diferença entre os dois grupos nem correlacionaram-se com as escalas do IDA TE-C, o que invalida tal escala para crianças da nossa população. Defende-se a criação de uma escala própria para crianças.
\end{abstract}

Palavras-chave: ansiedade, desenho da figura humana, validade.

\section{Is the human figure drawing a valid measure for children's ansiety assessment?}

\section{Summary}

Anxiety assessment has been one ofthe uses ofHuman Figure Drawing Test (HFD). The Handler HFD rating scale for anxiety, an important scoring system in this field, has generated several divergent studies about its validity. The main goal of this study was to investigate the validity of Handler indexes through an experimental model comparing HFD of 53 pediatric elective surgery patients, from 7 to 13 years old, to a control group. Results showed significant difference between experimental and control groups in tenns of STAI-C but no differences in tenns od HFD. This data, associated with the lack of significant correlation between STAI-C and HFD, suggest that Handler rating scale is not valid for our children.

Key words: anxiety, human figure drawing, validity.

\section{Introdução}

A ansiedade tem sido o objeto de um grande número de estudos, devido a sua relação com campos diversos abordados pela Psicologia. A ansiedade, conforme May (1977) conceitua, é um

\footnotetext{
1 Endereço para correspondência: Rua, Ramiro Barcelos. 2600. sala 120. CEP 90035-003, Porto Alegre - RS. E-mail bandeira@vortex.ufrgs.br. Os autores agradecem a colaboração da bolsista Angélica Giacomel.

${ }^{2}$ Professora Assistente do Instituto de Psicologia da Universidade Federal do Rio Grande do Sul.

${ }^{3}$ Psicóloga, ex-bolsista de Iniciação Científica pela FAPERGS.

${ }^{4}$ Anestesiologista do Hospital de Clínicas de Porto Alegre e Mestre em Medicina.

${ }^{5}$ Professora Adjunta do Instituto de Ciências Básicas da Saúde da Universidade Federal do Rio Grande do Sul.
} 
termo que se refere a uma relação de impotência, conflito existente entre a pessoa e o ambiente ameaçador, e os processos neurofisiológicos decorrentes dessa relação. O mesmo autor diz ainda que a ansiedade constitui a experiência subjetiva do organismo numa condição catastrófica, que surge na medida em que o indivíduo, frente a uma situação, não pode fazer face às exigências do seu meio, e, portanto, sente uma ameaça à sua existência ou aos valores que considera essenciais.

Uma das áreas onde esse tipo de estudo traz dados importantes para a melhoria da qualidade de vida é a Psicologia Hospitalar. A ansiedade prévia a uma situação cirúrgica tem sido estudada por diversos autores. Johnston (1987) constatou que a principal preocupação nesses casos reside no resultado da operação mais do que no procedimento em si mesmo. Shrimali \& Broota (1989) já demonstraram a relação entre o período antecedente à operação e a ansiedade, com os sujeitos à espera de cirurgia maiores demonstrando um nível de ansiedade maior do que o de grupos de cirurgias mais leves e de controle. Observaram, também, respostas afetivas tais como irritabilidade, apatia, depressão, raiva e desesperança com queda considerável após a cirurgia, que se somaram a grande desorientação cognitiva, que também declinou após a operação.

O Inventário de Ansiedade Traço-Estado (IDATE), de Spielberger, traduzido e adaptado para o Brasil por Biaggio $(1979,1983)$ é uma das formas utilizadas para avaliar a ansiedade. A ansiedade estado é um estado emocional transitório ou condição do organismo humano, caracterizado por sentimentos desagradáveis de tensão e apreensão conscientemente percebidos e por aumento na atividade do sistema nervoso autônomo. Já o traço de ansiedade refere-se às crenças individuais relativamente estáveis em propensão à ansiedade, isto é, diferença na tendência de reagir a situações percebidas como ameaçadoras com elevações de intensidade no estado de ansiedade (Biaggio, 1984). Segundo Endler, Parker, Bagby \& Cox (1991), a escala de ansiedade traço do IDATE parece avaliar a ansiedade primariamente em resposta a situações ameaçadoras ao ego, devido a uma tendência estável, e não parece modificar-se em outros tipos de situações ameaçadoras como aquelas que envolvem perigo físico.

Há evidências de que a ansiedade estado, por sua vez, possui dois componentes: reações cognitivas, que focam inadequações ou fracassos em potencial, e as reações autônomas ou emocionais, relacionadas com o funcionamento do sistema nervoso simpático (Endler, Parker, Bagby \& Cox, 1991). Isso deve ser evidenciado na situação de espera da cirurgia, sendo avaliado com o IDATE a ansiedade estado como unidimensional (Biaggio, 1984).

Situações de stress e ansiedade vêm sendo avaliadas também pelo Desenho da Figura Humana (DFH). Com a escala Goodenough-Harris de avaliação do DFH em crianças com câncer, constatou-se significativa redução na altura, largura e área em relação aos desenhos de crianças em escolas e pacientes de cirurgia geral, o que foi atribuído em parte à ansiedade (Paine, Alves \& Tubino, 1985).

Outra escala de avaliação do DFH, esta objetivando investigar a ansiedade, foi idealizada por Handler (1967). Os índices de ansiedade do DFH propostos por ele são, dentre diversos sistemas de escores, os que receberam principal atenção das pesquisas, conforme revisão de literatura específica sobre a medida de ansiedade através do desenho (Sims, Dana \& Bolton, 1983). A sua base está, em parte, nos trabalhos de Hoyt, 1955, e de Bolton, 1950 (citados em Handler, 1967). Handler introduziu modificações, preocupando-se em estabelecer critérios de escore para a análise de maneira formal, que abrangem tanto a ansiedade causada por situações externas estressantes como por causas intrafísicas. Vinte índices foram descritos, atribuindo-se escores de acordo com as 
características do desenho para cada um deles, em escalas de quatro ou dois pontos onde, nesta última, a presença é um indicador de ansiedade.

A escala de Handler foi elaborada para a avaliação da ansiedade nos desenhos de adolescentes e adultos, contudo seu sistema de avaliação alcançou alta correlação na aplicação da escala em crianças (Sopchak, 1970, Van Ko1ck, 1973). Ainda assim, são encontradas críticas à validade dessa escala e mesmo da utilização do DFH como instrumento diagnóstico de ansiedade (Engle e Suppes, 1970; Goldstein e Faterson, 1969; Handler, 1984; Sims, Dana, \& Bolton, 1983).

Com o objetivo verificar a validade dos índices de ansiedade da escala de Handler (1967) para a nossa população, idealizou-se o presente estudo. Neste, desenhos de crianças em situação pré-cirúrgica foram comparados a desenhos realizados por crianças de um grupo controle. Além disso, os pontos obtidos nos desenhos foram comparados aos escores no IDA TE-C, que serviu como mais uma fonte de investigação da ansiedade.

\section{Método}

Sujeitos

A amostra foi composta de dois grupos de 53 crianças cada, de sete a treze anos e ambos os sexos. $\mathrm{O}$ primeiro grupo foi composto por 3 I meninos e 22 meninas submetidos a procedimentos anestésico-cirúrgicos no Bloco Cirúrgico ou no Centro de Atendimento Ambulatorial do Hospital de Clínicas de Porto Alegre. A idade média das crianças deste grupo era de 121,19 meses, correspondente a 10 anos e I mês. O segundo grupo, de controle, foi composto por 29 meninos e 24 meninas, selecionado em escolas públicas de Porto Alegre. A idade média deste grupo ficou em 112,15, o equivalente a 10 anos e 2 meses.

\section{Instrumentos}

Para avaliar o nível de ansiedade das crianças foram utilizados o Inventário de Ansiedade Traço-Estado para Crianças (IDA TE-C), em sua adaptação para o Brasil por Biaggio (1983), e os indicadores de ansiedade do Desenho da Figura Humana propostos por Handler (1967). Para o DFH, foi utilizada uma folha de tamanho ofício, um lápis n 2 e uma borracha. A escala utilizada no levantamento dos indicadores de ansiedade de Handler foi a de dois pontos (ausente-presente) para os 18 primeiros índices. Os $19^{\circ}$ e $20^{\circ}$ índices não foram considerados, devido à grande dificuldade para aplicação e levantamento.

\section{Procedimentos}

Nas crianças do grupo experimental, os dois testes foram aplicados durante o período précirúrgico na sala de preparo do Bloco Cirúrgico ou do Centro de Atendimento Ambulatorial do Hospital de Clínicas. Nas crianças do grupo de controle, os testes foram aplicados individualmente ou em pequenos grupos (até quatro sujeitos), em salas das próprias escolas.

Os testes foram aplicados por alunos de Psicologia especialmente treinados em aplicação de testes, sendo que o DFH foi o primeiro instrumento aplicado com o intuito de que a criança se adaptasse à tarefa e, logo após, o IDATE-C foi aplicado. Para as crianças com dificuldade de entendimento do IDATE-C, houve auxílio das aplicadoras.

O levantamento do DFH foi realizado também por alunos de Psicologia treinados pela primeira autora, cuja correlação ficou em $r=0,862$, índice considerado bem elevado para escalas que envolvem componentes de avaliação por vezes subjetivos. 


\section{Resultados}

As médias obtidas no IDATE-C (ansiedade traço e ansiedade estado) e na escala de Handler (1967) para ambos os grupos (experimental e de controle) estão expostas na Tabela 1.

Tabela 1. Média e desvio-padrão dos escores brutos obtidos nas escalas de ansiedade traço e ansiedade estado do IDATE-C e no DFH nos grupos experimental e controle

\begin{tabular}{c|c|c}
\hline Teste & Grupo Experimental & Grupo Controle \\
\hline Ansiedade traço & $38,60(\mathrm{dp}=7.341$ & $35.89(\mathrm{dp}=7.041$ \\
\hline Ansiedade estado & $31.36(\mathrm{dp}=7.041$ & $27,23(\mathrm{dp}=4.75)$ \\
\hline Total de índices DF" & $7.94(\mathrm{dp}=2.71)$ & $7,23(\mathrm{dp}=2.71)$ \\
\hline
\end{tabular}

As análises de variância realizadas não indicaram diferenças entre os sexos $[\mathrm{F}(1,104)=1,794$ e $\mathrm{p}<0,183$ para a ansiedade traço, $\mathrm{F}(1,104)=0,243$ e $\mathrm{p}<0,623$ para ansiedade estado e $\mathrm{F}(1,104)=$ 0,168 e p<0,683 para o total de índices no DFH]. Portanto, todas as análises seguintes foram realizadas com o grupo total de sujeitos.

Entre os grupos experimental e controle, observaram-se diferenças significativas para a ansiedade traço $[\mathrm{F}(1,104)=3,866$ e $\mathrm{p}<0,052]$ e a ansiedade estado $[\mathrm{F}(1,104)=12,557$ e $\mathrm{p}<0,001]$. Contudo, para o total de índices de ansiedade do $\mathrm{DFH}$, não foram verificadas diferenças significativas $[\mathrm{F}(1,104)=2,315$ e $\mathrm{p}<0,131]$.

Outro dado relevante diz respeito às correlações entre as escalas ansiedade traço estado do IDATE-C e índices de ansiedade do DFH. Tais resultados encontram-se na Tabela 2, indicando que somente há correlação entre as escalas do IDA TE-C, resultado já esperado conforme Biaggio (1983).

Tabela 2. Correlações entre as escalas de ansiedade traço e ansiedade estado do IDA TE-C e de ansiedade do DFH

\begin{tabular}{|c|c|c|c|}
\hline & Ansiedade traço & Ansiedade estado & Ansiedade DF" \\
\hline Ansiedade traço & 1.000 & $0.393^{*}$ & 0,246 \\
\hline Ansiedade estado & $0.393^{*}$ & 1,000 & 0.010 \\
\hline Ansiedade DF" & 0.246 & 0.010 & 1.000 \\
\hline
\end{tabular}

* Correlações significativas a $\mathrm{p}<0,01$

\section{Discussão}

Os dados encontrados confirmam achados que indicam que crianças em situação précirúrgica apresentam níveis de ansiedade mais elevados (Biaggio, 1984). Neste tipo de situação, onde a ameaça física é mais presente, esperar-se-ia aumento apenas na ansiedade estado, conforme Endler, Parker, Bagby \& Cox (1991). Neste estudo, ambas as escalas de ansiedade do IDATE-C apresentaram diferenças entre os grupos. Contudo, é de se supor que uma cirurgia também possa representar, em fantasia, uma ameaça ao ego para a criança. Biaggio (1984) também percebeu aumento da ansiedade traço de crianças em situações ansiogênicas. 
Analisando-se os resultados comparativos do escore total dos índices do DFH entre os dois grupos, percebe-se que as médias encontradas são muito semelhantes (Tabela I). Este dado, aliado à falta de correlação entre tal escore e as escalas de ansiedade traço-estado, indicam a não validade do sistema de levantamento de Handler (1967) para crianças na nossa população. Uma análise individual dos índices permitiu ainda mostrar que apenas um deles correlaciona-se significativamente $(r=0,251$ a $\mathrm{p}<0,009)$ com o IDA TE-C e esta correlação se dá com a escala de ansiedade-estado. Este índice avalia a interrupção de linhas e aparece em percentual igual nos dois grupos (três entre 53 casos), indicando que a correlação pode ter sido um mero acaso.

\section{Conclusão}

Os achados deste trabalho vão ao encontro das críticas apresentadas por outros autores a este sistema de levantamento, realizadas inclusive pelo próprio Handler (1984) e outros pesquisadores (Engle e Suppes, 1970; Goldstein e Faterson, 1969; Sims, Dana, \& Bolton, 1983). Apesar de ser um dos mais bem descritos manuais de levantamento do DF H, com um nível de detalhamento elevado, peca no que diz respeito à investigação de ansiedade em crianças da nossa população.

É provável que o nível de exigência dessa escala iguale as crianças em termos de maturidade. Em nível de ilustração, o $17^{\circ}$ índice, por exemplo, busca identificar o nível de simplificação da cabeça. Grande parte das crianças, independente de estarem ansiosas ou não, vai virtualmente pontuar neste item, tendo em vista sua falta de maturidade para conseguir desenhar uma cabeça em formato oblongo e feições (olhos, nariz, boca) compostas por mais do que linhas e de formato não circular. Ou ainda o índice $18^{\circ}$ onde para não pontuar, a criança precisa desenhar os braços adequadamente ligados ao tronco. Em seu manual para crianças brasileiras, Wechsler (1996) demonstra que a presença de olhos mais compridos que redondos, por exemplo, é incomum (de 16 a 50\%) em crianças de 10 anos. Em um trabalho sobre normas para o DFH com crianças brasileiras, Hutz e Antoniazzi (1995) mostram que a presença de braços adequadamente ligados ao corpo é excepcional para crianças até 7 anos, não aparecendo como item esperado para crianças de 7 a 15 anos.

Diante disso, seria necessário que se construísse uma escala própria para crianças, com bons descritores, já que o DFH continua sendo um instrumento largamente utilizado na prática e na pesquisa psicológica (Hutz e Bandeira, 1993; Lubin, Walls, Matarazzo e Seever, 1985).

\section{Referências}

Biaggio, A. M. B. (1979). Manual do Inventário de Ansiedade Traço-Estado (IDATE). Rio de Janeiro, RJ: CEPA.

Biaggio, A. M. B. (1983). Manual do Inventário de Ansiedade Traço-Estado para crianças (IDATE-C). Rio de Janeiro, RJ: CEPA.

Biaggio, A. M. B. (1984). Pesquisas em Psicologia do desenvolvimento e da personalidade. Porto Alegre, RS: Editora da Universidade-UFRGS.

Endler, N.; Parker, 1.; Bagby, R. \& Cox, B. (1991). Multidimensionality of state and trait anxiety: factar structure of the Endler Multidimensional Anxiety Scales. Journal of Personality and Social Psychology, $60,919-926$. 
Engle, P. L., \& Suppes, J. S. (1970). The relation between human figure drawing and test anxiety in children. Journal of Projective Techniques and Personality Assesment. 34, 223-231.

Handler, L. (1967). Anxiety indexes in the Draw-a Person Test; a scoring manual. Journal of Projective Techniques and Personality Assesment. 31,46-57.

Handler, L. (1984). Anxiety as measured by the Draw-a-Person Test: a response to Sims, Dana, and Bolton. Journal of Personality Assesment, 48, 82-84.

Hutz, C. S., \& Antoniazzi, A. S. (1995). O desenvolvimento do Desenho da Figura Humana em crianças de 5 a 15 anos de idade: Normas para avaliação. Psicologia: Reflexão e Crítica. 8,3-18.

Hutz, C. S., \& Bandeira, D. R. (1993). Tendências contemporâneas no uso de testes: Uma análise da literatura brasileira e internacional. Psicologia: Reflexão e Crítica, 6, 85-101.

Goldstein, H. S., \& Faterson, H. F. (1969). Shading as an index of anxiety in figure drawings. Journal of Projective Techniques \& Personality Assessment, 33,454-456.

Johnston, M. (1987). Emotional and Cognitive aspects of anxiety in surgical patients. Communication \& Cognition, 20(3),245-260.

Lubin, B., Walls, R., Matarazzo, J. D., \& Seever, M. (1985). Psychological test usage patterns in five professional settings. American Psychologist. 40, 857-861.

May, R. (1977). O significado da ansiedade. Rio de Janeiro: Zahar.

Paine, P.; Alves, E. \& Tubino, P. (1985). Size of human figure drawing and Goodenough-Harris scores on pediatric oncology patients: A pilot study. Perceptual and Motor Skills, 60, 911-914.

Shrimali, S. \& Broota, K. D. (1989). Perception regarding the surgeon as a function of surgical stress. Journal of Personality \& Clinical Studies, 5, 211-215.

Sims, 1.; Dana, R. \& Bolton, B. (1983). The validity of the Draw a Person Test as an Anxiety Measure. Journal of Personality Assesment, 47(3),250-257.

Sopchak, A. L. (1970). Anxiety indicators on the Draw-a- Person Test for c1inic and nonclinic boys and their parents. Journal of Psychology. 76, 251-260.

Van Kolck, O. L. (1973). Sinais de ansiedade e de distúrbios emocionais no desenho da figura humana de crianças - tentativa de validação. Boletim de Psicologia, 65, 11-45.

Wechsler, S. M. (1996). O desenho da figura humana: Avaliação do desenvolvimento cognitivo infantil. Manual para crianças brasileiras. Campinas, SP: Editorial Psy. 